\title{
ON OPTIMAL CLOAKING-BY-MAPPING TRANSFORMATIONS
}

\author{
Yves CapdeboscQ ${ }^{1, *}$ (1) And Michael S. Vogelius ${ }^{2}$ (i)
}

\begin{abstract}
A central ingredient of cloaking-by-mapping is the diffeomorphism which transforms an annulus with a small hole into an annulus with a finite size hole, while being the identity on the outer boundary of the annulus. The resulting meta-material is anisotropic, which makes it difficult to manufacture. The problem of minimizing anisotropy among radial transformations has been studied in Griesmaier and Vogelius [Inverse Prob. 30 (2014) 17]. In this work, as in Griesmaier and Vogelius [Inverse Prob. 30 (2014) 17], we formulate the problem of minimizing anisotropy as an energy minimization problem. Our main goal is to provide strong evidence for the conjecture that for cloaks with circular boundaries, non-radial transformations do not lead to lower degree of anisotropy. In the final section, we consider cloaks with non-circular boundaries and show that in this case, non-radial cloaks may be advantageous, when it comes to minimizing anisotropy.
\end{abstract}

Mathematics Subject Classification. 35R30, 78A46.

Received September 6, 2021. Accepted January 5, 2022.

\section{INTRODUCTION}

A central ingredient in the construction of (approximate) cloaks by the passive cloaking technique, known as "cloaking by mapping", is the diffeomorphism, which transforms an annulus with a small hole into an annulus with a finite size hole, and which is the identity on the outer boundary of the annulus. The pushforward of the background coefficient (say, the identity matrix) with the diffeomorphism represents the metamaterial needed for the cloak, and the finite size hole is the area that may be used as a "hiding place" [8]. The fact that the diffeomorphism is the identity on the outer boundary ensures that the perturbation in the "far field" is that corresponding to a small inhomogeneity. The corresponding "lack of cloaking"/visibility can be estimated by the volume of the small inhomogeneity. The required meta-material is anisotropic, which presents a problem when it comes to actual manufacture of the cloak. Typically a radial affine transformation has been used $[4,5,8-10]$, however, a very natural question arises, namely : "are there transformations that lead to lower degree of anisotropy than the radial affine transformation?" In [6] it was shown that there are indeed better radial transformations than the affine, when it comes to minimizing anisotropy. In that paper the meta-material obtained by "optimal radial transformation" is also shown to be quite related to meta-materials obtained by other cloak enhancement strategies, employing additional layers [3, 7]. The focus of this note is to produce very strong evidence for the conjecture that when the cloak takes the shape of

Keywords and phrases. Cloak enhancement, approximate cloaking, cloaking-by-mapping, optimal cloaking.

1 Université de Paris, CNRS, Sorbonne Université, Laboratoire Jacques-Louis Lions (LJLL), F-75006 Paris, France.

2 Department of Mathematics, Rutgers University, New Brunswick, NJ 08901, USA.

*Corresponding author: yves. capdeboscq@u-paris.fr

(C) The authors. Published by EDP Sciences, SMAI 2022

This is an Open Access article distributed under the terms of the Creative Commons Attribution License (https://creativecommons.org/licenses/by/4.0), which permits unrestricted use, distribution, and reproduction in any medium, provided the original work is properly cited. 
a classical annulus, non-radial transformations do not help in reducing the degree of anisotropy. Like in [6], we formulate the problem of minimizing anisotropy as a variational problem (minimization of an appropriate energy). Corollary 4.4 summarizes our main results. Broadly speaking, we show that

- There exists a radial transformation, which is a stationary point for the energy.

- This radial transformation has smaller energy than all other transformations with "directional field" $\frac{x}{|x|}$.

- If the amplitude is kept fixed and radial, then any change in the "directional field" away from $\frac{x}{|x|}$ will increase energy.

In the final section of this note we consider the case when the outer (and inner) boundary of the cloak are not circles, and we illustrate how the optimal radial transformation for the circular case translates into a non-radial (optimal) transformation for a non-circular cloak.

\section{Preliminaries}

For $r>0$ we set

$$
B_{r}=\left\{x \in \mathbb{R}^{2}:|x|<r\right\}, \text { and } C_{r}=\left\{x \in \mathbb{R}^{2}:|x|=r\right\} .
$$

Given $0<\epsilon<1 / 2$, we shall use the notation $\Phi$ for a bijective diffeomorphism $\overline{B_{1}} \backslash B_{\epsilon} \rightarrow \overline{B_{1}} \backslash B_{\frac{1}{2}}$ with $\Phi \in C^{1}\left(\overline{B_{1}} \backslash B_{\epsilon} ; \overline{B_{1}} \backslash B_{\frac{1}{2}}\right)$, and $\Phi^{-1} \in C^{1}\left(\overline{B_{1}} \backslash B_{\frac{1}{2}} ; \overline{B_{1}} \backslash B_{\epsilon}\right)$. We furthermore impose that

$$
\left.\Phi\right|_{C_{1}}=I d, \text { and } \Phi\left(C_{\epsilon}\right)=C_{\frac{1}{2}} \text {. }
$$

One such transformation is the radial affine transformation, given by

$$
x \rightarrow\left(\frac{|x|-1}{2(1-\epsilon)}+1\right) \frac{x}{|x|} .
$$

The push-forward of the identity matrix with the diffeomorphism $\Phi$ is given by

$$
\Phi *[I](\Phi(x))=\frac{D \Phi D \Phi^{T}}{|\operatorname{det} D \Phi|}(x) .
$$

This is a positive definite matrix, and since we are in two dimensions, with determinant 1 . Let $0<\lambda_{1}(x) \leq$ $1 \leq \lambda_{2}(x)$ denote the eigenvalues of $\Phi *[I](\Phi(x))$. A natural measure of the degree of anisotropy of $\Phi *[I]$ at the point $\Phi(x)$ is

$$
\begin{aligned}
\left|\lambda_{1}(x)-1\right|+\left|\lambda_{2}(x)-1\right|=\lambda_{2}(x)-\lambda_{1}(x) & =\sqrt{\left(\lambda_{2}(x)-\lambda_{1}(x)\right)^{2}} \\
& =\sqrt{\left(\lambda_{1}(x)+\lambda_{2}(x)\right)^{2}-4}
\end{aligned}
$$

To minimize this we must minimize trace $\Phi_{*}[I](\Phi(x))$. As a way of minimizing the aggregate anisotropy we shall seek to minimize ${ }^{1}$

$$
I_{p}(\Phi)=\int_{B_{1} \backslash B_{\epsilon}}(\operatorname{trace} \Phi *[I])^{p}(\Phi(x)) \mathrm{d} x
$$

for a fixed choice of $1 \leq p<\infty$, and

$$
I_{\infty}(\Phi)=\max _{x \in \overline{B_{1} \backslash B_{\epsilon}}} \operatorname{trace} \Phi *[I](\Phi(x))=\max _{y \in \overline{B_{1}} \backslash B_{\frac{1}{2}}} \operatorname{trace} \Phi *[I](y)
$$

\footnotetext{
${ }^{1}$ In a slight deviation from [6], the domain of integration of the energy functional is $B_{1} \backslash B_{\epsilon}$, not the transformed domain $B_{1} \backslash B_{\frac{1}{2}}$.
} 
corresponding to $p=\infty$. Note that $\lambda$ is an eigenvalue for $\Phi_{*}[I](\Phi(x))$, with eigenvector $v$, if and only if $\lambda$ is an eigenvalue for

$$
\frac{D \Phi^{T} D \Phi}{|\operatorname{det} D \Phi|}(x)
$$

with eigenvector $D \Phi^{T}(x) v$, and thus

$$
\operatorname{trace} \Phi_{*}[I](\Phi(x))=\operatorname{trace}\left[\frac{D \Phi^{T} D \Phi}{|\operatorname{det} D \Phi|}\right](x) .
$$

Proposition 2.1. Let $\Phi$ be represented in terms of its polar decomposition

$$
\Phi=\exp (\psi) \phi
$$

where the directional field $\phi$ is in $C^{1}\left(\overline{B_{1}} \backslash B_{\epsilon} ; \mathcal{S}^{1}\right)$ and the logarithmic amplitude $\psi$ is in $C^{1}\left(\overline{B_{1}} \backslash B_{\epsilon} ; \mathbb{R}\right)$. Then

$$
\operatorname{trace}\left(D \Phi^{T} D \Phi\right)=|\Phi|^{2}\left(|D \phi|^{2}+|D \psi|^{2}\right) \text {. }
$$

Proof. Differentiating we find

$$
D \Phi=\exp (\psi) \phi D \psi^{T}+\exp (\psi) D \phi
$$

Since $\phi^{T} \phi=1$, we have

$$
\phi^{T} D \phi=0, \text { and } D \phi^{T} \phi=0,
$$

and therefore

$$
\begin{aligned}
D \Phi^{T} D \Phi & =\exp (2 \psi)\left(D \psi \phi^{T}+D \phi^{T}\right)\left(\phi D \psi^{T}+D \phi\right) \\
& =|\Phi|^{2}\left(D \phi^{T} D \phi+D \psi D \psi^{T}\right) .
\end{aligned}
$$

By taking the trace we arrive at the desired conclusion.

It is well known that $\phi$, being in $C^{1}\left(\overline{B_{1}} \backslash B_{\epsilon} ; \mathcal{S}^{1}\right)$, admits a canonical lift $\theta=\arg (\phi) \in C^{1}\left(\overline{B_{1}} \backslash B_{\epsilon} ; \mathbb{R} / 2 \pi \mathbb{Z}\right)^{2}$ such that

$$
\phi=(\cos \theta, \sin \theta)^{T} \text {. }
$$

We write

$$
J=\left[\begin{array}{cc}
0 & -1 \\
1 & 0
\end{array}\right], \quad \mathrm{e}_{r}=\frac{x}{|x|}, \quad \text { and } \quad \mathrm{e}_{\theta}=J \frac{x}{|x|} .
$$

Proposition 2.2. The matrix $D \phi$ has rank one; furthermore

$$
\operatorname{Range}(D \phi)=\operatorname{Span}(\phi)^{\perp} \text {, and } \operatorname{Ker}(D \phi)=\operatorname{Span}(D \theta)^{\perp} .
$$

We denote by $\widehat{D \psi, D} \theta$ the angle defined by

$$
\begin{aligned}
& \cos (\widehat{D \psi, D} \theta)=\frac{1}{|D \psi||D \theta|} D \psi \cdot D \theta, \quad \text { and } \\
& \sin (\widehat{D \psi, D} \theta)=\frac{1}{|D \psi||D \theta|} \operatorname{det}(D \psi, D \theta) .
\end{aligned}
$$

Then

$$
\operatorname{trace} \Phi *[I](\Phi(x))=\frac{1}{|\sin (\widehat{D \psi, D} \theta)|}\left(\frac{|D \theta|}{|D \psi|}+\frac{|D \psi|}{|D \theta|}\right)(x) \geq\left(\frac{|D \theta|}{|D \psi|}+\frac{|D \psi|}{|D \theta|}\right)(x)
$$

with equality only when $D \psi \cdot D \theta=0$.

\footnotetext{
${ }^{2} \mathrm{~A}$ function $\theta: \overline{B_{1}} \backslash B_{\epsilon} \rightarrow \mathbb{R} / 2 \pi \mathbb{Z}$ is an element of $C^{1}\left(\overline{B_{1}} \backslash B_{\epsilon} ; \mathbb{R} / 2 \pi \mathbb{Z}\right)$ iff given any point $x \in \overline{B_{1}} \backslash B_{\epsilon}$ there exists an open neighborhood $\omega_{x}$ of $x$, relative to $\overline{B_{1}} \backslash B_{\epsilon}$, and a representative of $\theta(\bmod 2 \pi)$ that lies in $C^{1}\left(\omega_{x} ; \mathbb{R}\right)$. Notice that the globally defined derivative of $\theta \in C^{1}\left(\overline{B_{1}} \backslash B_{\epsilon} ; \mathbb{R} / 2 \pi \mathbb{Z}\right), D \theta$, lies in $C^{0}\left(\overline{B_{1}} \backslash B_{\epsilon} ; \mathbb{R}^{2}\right)$.
} 
Proof. We calculate

$$
D \phi=(J \phi) D \theta^{T},
$$

which immediately leads to the statements about $\operatorname{Range}(D \phi)$ and $\operatorname{Ker}(D \phi)$, and which also gives

$$
D \theta=(D \phi)^{T}(J \phi)
$$

As a consequence

$$
\begin{aligned}
\operatorname{det} D \Phi & =\operatorname{det}\left(\phi D \psi^{T}+(J \phi) D \theta^{T}\right) \exp (2 \psi) \\
& =\operatorname{det}(D \psi, D \theta)|\Phi|^{2} \\
& =|D \theta||D \psi| \sin (\widehat{D \psi, D} \theta)|\Phi|^{2} .
\end{aligned}
$$

Here we have used that $\operatorname{det} D \Phi \neq 0$, since $\Phi$ is a bijective diffeomorphism of $\overline{B_{1}} \backslash B_{\epsilon}$ onto $\overline{B_{1}} \backslash B_{\frac{1}{2}}$; consequently $\operatorname{det}(D \psi, D \theta) \neq 0$ and $|D \psi||D \theta|>0$ and $\sin (\widehat{D \psi, D} \theta$ ) (and $\widehat{D \psi, D} \theta)$ is well-defined. It now follows that

$$
\begin{aligned}
\operatorname{trace} \Phi *[I](\Phi(x)) & =\frac{\left(|D \theta|^{2}+|D \psi|^{2}\right)}{|D \theta||D \psi||\sin (\widehat{D \psi, D} \theta)|}(x) \\
& =\frac{1}{|\sin (\widehat{D \psi, D} \theta)|}\left(\frac{|D \theta|}{|D \psi|}+\frac{|D \psi|}{|D \theta|}\right)(x) \geq \frac{|D \theta|}{|D \psi|}(x)+\frac{|D \psi|}{|D \theta|}(x),
\end{aligned}
$$

with equality if and only of $D \psi$ is normal to $D \theta$, and therefore in the kernel of $D \phi$.

\section{The RADiAL TRANSFORMATION CASE}

For the general case of a radial transformation $\phi=\frac{x}{|x|}$, and $\psi=f(|x|)$. Then $D \theta=\frac{1}{|x|} J \frac{x}{|x|}$ and $D \psi=$ $f^{\prime}(|x|) \frac{x}{|x|}$. The transformation

$$
\Phi=\exp (\psi) \phi
$$

is a bijective $C^{1}$ diffeomorphism of $\overline{B_{1}} \backslash B_{\epsilon}$ onto $\overline{B_{1}} \backslash B_{\frac{1}{2}}$ with

$$
\left.\Phi\right|_{C_{1}}=I d, \text { and } \Phi\left(C_{\epsilon}\right)=C_{\frac{1}{2}}
$$

if and only if

$$
f(\epsilon)=-\log 2, f(1)=0, \text { and } f \in C^{1}([\epsilon, 1]) \text { with } f^{\prime}(r)>0 \text { for all } r \in[\epsilon, 1] .
$$

In this case, $\sin (\widehat{D \psi, D} \theta)=1$, and

$$
\operatorname{trace} \Phi *[I](\Phi(x))=\frac{1}{|x| f^{\prime}(|x|)}+|x| f^{\prime}(|x|) .
$$

Proposition 3.1. Suppose $1 \leq p<\infty$, and let $I_{p}$ denote the energy

$$
I_{p}(f):=\int_{B_{1} \backslash B_{\epsilon}}(\operatorname{trace} \Phi *[I])^{p}(\Phi(x)) \mathrm{d} x=2 \pi \int_{\epsilon}^{1}\left(\frac{1}{r f^{\prime}(r)}+r f^{\prime}(r)\right)^{p} r \mathrm{~d} r,
$$

with values in $(0, \infty)$, defined on the convex set

$$
\mathcal{C}=\left\{f \in C^{1}([\epsilon, 1]): f^{\prime}>0, f(\epsilon)=-\log 2, f(1)=0\right\} .
$$

Then 
- Ip has a unique minimizer, $f_{p}$, in $\mathcal{C}$.

- $f_{p}$ lies in $C^{\infty}([\epsilon, 1])$, and is the unique solution in $\mathcal{C}$ to the Euler-Lagrange equation

$$
\left(\left(\frac{1}{r f_{p}^{\prime}(r)}+r f_{p}^{\prime}(r)\right)^{p-1}\left(-\frac{1}{\left(f_{p}^{\prime}\right)^{2}}+r^{2}\right)\right)^{\prime}=0 \quad \text { in }[\epsilon, 1] .
$$

Proof. We start by establishing (part of) the last statement concerning the existence of a unique solution to the Euler-Lagrange equation (E-L). By integration, any $C^{1}$ solution to (E-L) must satisfy

$$
G\left(r f_{p}^{\prime}(r)\right)=\frac{C}{r^{2}}
$$

for some constant $C$, with the function $G: \mathbb{R}_{+} \rightarrow \mathbb{R}$ given by

$$
G(t)=\left(\frac{1}{t}+t\right)^{p-1}\left(-\frac{1}{t^{2}}+1\right)
$$

Now suppose $1<p<\infty$. A simple calculation shows that $G$ is monotonically increasing, with $G(1)=0$, $\lim _{t \rightarrow 0_{+}} G(t)=-\infty$ and $\lim _{t \rightarrow \infty} G(t)=\infty . G^{-1}: \mathbb{R} \rightarrow \mathbb{R}_{+}$is thus well defined, and $f_{p}$ has the form

$$
f_{p}(r)=\int_{\epsilon}^{r} f_{p}^{\prime}(t) \mathrm{d} t-\log 2=\int_{\epsilon}^{r} t^{-1} G^{-1}\left(\frac{C}{t^{2}}\right) \mathrm{d} t-\log 2,
$$

for some constant $C$. The constant $C$ must be chosen so that $f_{p}$ satisfies the boundary condition $f_{p}(1)=0$. As $C \rightarrow \int_{\epsilon}^{1} t^{-1} G^{-1}\left(\frac{C}{t^{2}}\right) \mathrm{d} t-\log 2$ is continuous and monotonically increasing, with

$$
\int_{\epsilon}^{1} t^{-1} G^{-1}\left(\frac{C}{t^{2}}\right) \mathrm{d} t-\log 2 \rightarrow \begin{cases}|\log \epsilon|-\log 2>0 & \text { when } C \rightarrow 0 \\ -\log 2<0 & \text { when } C \rightarrow-\infty\end{cases}
$$

it follows immediately that there exists a unique value $C_{0}<0$ for which the boundary condition $f_{p}(1)=0$ is satisfied. This shows the uniqueness of the solution to the Euler-Lagrange equation in $\mathcal{C}$. Furthermore, the formula

$$
f_{p}(r)=\int_{\epsilon}^{r} t^{-1} G^{-1}\left(\frac{C_{0}}{t^{2}}\right) \mathrm{d} t-\log 2
$$

clearly gives rise to a $C^{\infty}$ function in $\mathcal{C}$ which solves the equation (E-L), thus establishing the existence ${ }^{3}$. A slightly modified argument works for $p=1$, and in that case we find the (even more) explicit formula

$$
f_{1}: r \rightarrow \log \left(\frac{3 r+\sqrt{9 r^{2}+16(2-\epsilon)\left(\frac{1}{2}-\epsilon\right)}}{4(2-\epsilon)}\right) .
$$

We now proceed to show that $f_{p}$ is the unique minimizer of $I_{p}$ in $\mathcal{C}$. Since the function $(0, \infty) \ni x \rightarrow\left(\frac{1}{x}+x\right)^{p} \in$ $(0, \infty)$ is strictly convex, it follows immediately that $I_{p}$ is strictly convex on $\mathcal{C}$. Now suppose there existed a function $g \in \mathcal{C}$ with $I_{p}(g)<I_{p}\left(f_{p}\right)$. The convexity of the functional $I_{p}$ implies that

$$
\left.\frac{\mathrm{d}}{\mathrm{d} \tau}\right|_{\tau=0} I_{p}\left(f_{p}+\tau\left(g-f_{p}\right)\right) \leq I_{p}(g)-I_{p}\left(f_{p}\right)<0,
$$

${ }^{3}$ With $z_{p}(t)=G^{-1}\left(C / t^{2}\right), C<0$, one has the formula

$$
\int t^{-1} z_{p}(t)=\frac{1}{2} \log \frac{1+z_{p}(t)}{1-z_{p}(t)}+(p-1)\left(\arctan z_{p}(t)-\frac{1}{2} z_{p}(t)\right)+\text { const }
$$

for the indefinite integral of $z_{p}(t) / t$. This formula proves extremely useful when computing the $f_{p}$ 's, and in particular their $\epsilon \rightarrow 0$ limits, numerically. 
or

$$
\int_{\epsilon}^{1}\left(\frac{1}{r f^{\prime}(r)}+r f^{\prime}(r)\right)^{p-1}\left(-\frac{1}{\left(f_{p}^{\prime}\right)^{2}}+r^{2}\right)\left(g-f_{p}\right)^{\prime} \mathrm{d} r<0,
$$

in contradiction with the fact that $f_{p}$ is a solution to the Euler-Lagrange equation (E-L). This verifies that $f_{p}$ is a minimizer of $I_{p}$ in $\mathcal{C}$. The fact that the minimizer is unique follows immediately from the strict convexity of $I_{p}$.

Remark. The logarithmic amplitude $f_{1}$ gives rise to the transformation

$$
\Phi_{1}=\left(\frac{3|x|+\sqrt{9|x|^{2}+16(2-\epsilon)\left(\frac{1}{2}-\epsilon\right)}}{4(2-\epsilon)}\right) \frac{x}{|x|} .
$$

We compute

$$
I_{1}\left(f_{1}\right)=2 \pi \int_{\epsilon}^{1}\left(\frac{1}{f_{1}^{\prime}(r)}+r^{2} f_{1}^{\prime}(r)\right) \mathrm{d} r=2 \pi\left(1-\epsilon^{2}+\frac{2}{3}(2 \epsilon-1)^{2}\right) .
$$

By comparison, the radial affine transformation

$$
\Phi_{r a}=\left(\frac{|x|-1}{2(1-\epsilon)}+1\right) \frac{x}{|x|}
$$

with logarithmic amplitude

$$
f_{r a}(r)=\log \left(\frac{r-1}{2(1-\epsilon)}+1\right)
$$

has

$$
I_{1}\left(f_{r a}\right)=2 \pi \int_{\epsilon}^{1}\left(\frac{1}{f_{r a}^{\prime}(r)}+r^{2} f_{r a}^{\prime}(r)\right) \mathrm{d} r=2 \pi\left(1-\epsilon^{2}+\ln 2(2 \epsilon-1)^{2}\right) \geq I_{1}\left(f_{1}\right) .
$$

Equality occurs only when $\epsilon=\frac{1}{2}$ (when the associated transformations are both the identity).

Turning to maximum norm, we consider the minimization

$$
\mathcal{I}_{\infty}=\inf _{f \in \mathcal{C}} \sup _{[\epsilon, 1]}\left(\frac{1}{r f^{\prime}(r)}+r f^{\prime}(r)\right) .
$$

We note that

$$
\begin{aligned}
\mathcal{I}_{\infty} & =\inf _{K>1}\left\{\frac{1}{K}+K: \exists f \in \mathcal{C} \text { with } \sup _{r \in[\epsilon, 1]}\left\{\frac{1}{r f^{\prime}(r)}+r f^{\prime}(r)\right\} \leq \frac{1}{K}+K\right\} \\
& \geq \inf _{K>1}\left\{\frac{1}{K}+K: \exists f \in \mathcal{C} \text { with } \frac{1}{K}|\log r| \leq|f(r)|\right\} \\
& \geq \inf \left\{\frac{1}{K}+K: \frac{|\log \epsilon|}{\log 2} \leq K\right\}=\frac{\log 2}{|\log \epsilon|}+\frac{|\log \epsilon|}{\log 2}
\end{aligned}
$$

Here we have used that, if $f \in \mathcal{C}$ and if $K>1$, then

$$
\begin{aligned}
\frac{1}{r f^{\prime}(r)}+r f^{\prime}(r) \leq \frac{1}{K}+K \text { in }(\epsilon, 1) & \Longrightarrow \frac{1}{K r} \leq f^{\prime}(r) \leq \frac{K}{r} \text { in }(\epsilon, 1) \\
& \Longrightarrow \frac{1}{K}|\log r| \leq|f(r)| \leq K|\log r| \text { in }(\epsilon, 1) .
\end{aligned}
$$


On the other hand, the function

$$
f_{\infty}(r)=\frac{\log 2}{|\log \epsilon|} \log r
$$

lies in $\mathcal{C}$, and has $I_{\infty}\left(f_{\infty}\right)=\frac{\log 2}{|\log \epsilon|}+\frac{|\log \epsilon|}{\log 2}$.

It now follows immediately that $f_{\infty}$ is a minimizer of $I_{\infty}$ in $\mathcal{C}$. The following graph shows the amplitudes $\exp f_{r a}$ (dashed orange line), $\exp f_{1}, \exp f_{2}, \exp f_{3}, \exp f_{5}, \exp f_{8}, \exp f_{13}$ and $\exp f_{\infty}$ (solid lines from red to green), for $\epsilon=1 / 1000$. For illustration we have included the limiting amplitude of $\exp f_{3}$ as $\epsilon$ tends to zero. This is shown as a dotted line next to $\exp f_{3}$.

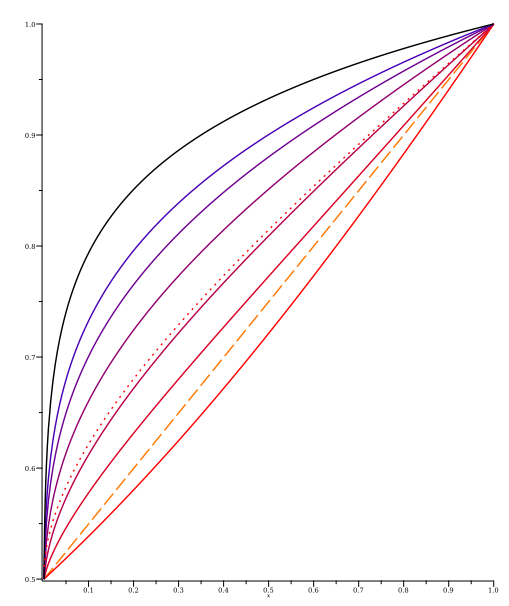

The optimal radial map for the $L^{\infty}$ norm given by

$$
\Phi_{\infty}: x \rightarrow \exp f_{\infty}(|x|) \frac{x}{|x|}=|x|^{\frac{\log 2}{|\log \epsilon|}-1} x
$$

is very similar to the one computed in [6] for a slightly different criterion. It is well approximated by one of the radial changes of variable suggested in [11] for an appropriate parameter choice. The maps associated with all these amplitudes are radial, but the materials obtained by push-forward transformations are not isotropic. A number of articles have investigated how to best achieve similar cloaking effects with layers of isotropic materials $[1-3,6,7,11]$. In this context we note that, it is well known from homogenization theory that the field responses of effective anisotropic impedance matrices are well approximated by those arising from constructs based on multiple fine isotropic laminates. The $L^{p}$ optimal maps $\Phi_{p}(x)=\exp f_{p}(|x|) \frac{x}{|x|}, 1 \leq p<\infty$ (just as $\Phi_{r a}$ ) have non-trivial limits as $\epsilon \rightarrow 0$, corresponding to maps of the punctured unit disk to the annulus $\overline{B_{1}} \backslash B_{1 / 2}$. However, the map $\Phi_{\infty}$ degenerates as $\epsilon \rightarrow 0$, as it approaches $\frac{x}{|x|}$, except for a vanishing boundary layer. The $L^{p}$ optimal maps $\Phi_{p}, 1 \leq p<\infty$, to the best of our knowledge, do not appear in other works.

\section{Optimality OF RADiAL TRANSFORMS}

We now return to the general, two dimensional case. By introducing $u=\psi$ and $V=-J D \theta$ in the formula

$$
\operatorname{trace} \Phi *[I](\Phi(x))=\frac{|D \psi|^{2}+|D \theta|^{2}}{\operatorname{det}(D \psi, D \theta)}(x),
$$


we obtain

$$
\operatorname{trace} \Phi *[I](\Phi(x))=\frac{|D u|^{2}+|V|^{2}}{D u \cdot V}(x) .
$$

Similarly, by introducing $u=\theta$ and $V=J D \psi$, we obtain

$$
\operatorname{trace} \Phi *[I](\Phi(x))=\frac{|D u|^{2}+|V|^{2}}{D u \cdot V}(x) .
$$

We thus notice that the problem of minimizing

$$
I_{p}(\Phi)=\int_{B_{1} \backslash B_{\epsilon}}(\operatorname{trace} \Phi *[I])^{p}(\Phi(x)) \mathrm{d} x
$$

with respect to $\psi$ given $\theta$, and with respect to $\theta$, given $\psi$ merely differs by a change of the convex test set for $u$ (essentially relating to boundary conditions). Let $\arg \in C^{\infty}\left(\overline{B_{1}} \backslash B_{\epsilon} ; \mathbb{R} / 2 \pi \mathbb{Z}\right)^{4}$ denote the standard argument function. We introduce the convex sets

$$
\begin{aligned}
& \mathcal{C}_{\theta}=C^{2, \alpha}\left(\overline{B_{1}} \backslash B_{\epsilon} ; \mathbb{R} / 2 \pi \mathbb{Z}\right) \cap\left\{\left.u\right|_{C_{1}}=\arg \right\} \text { and } \\
& \mathcal{C}_{\psi}=C^{2, \alpha}\left(\overline{B_{1}} \backslash B_{\epsilon} ; \mathbb{R}\right) \cap\left\{\left.u\right|_{C_{\epsilon}}=-\log 2,\left.u\right|_{C_{1}}=0\right\},
\end{aligned}
$$

for some fixed $\alpha>0$.

Proposition 4.1. Given $\mathcal{C}=\mathcal{C}_{\psi}$ and a fixed $V \in C^{0}\left(\overline{B_{1}} \backslash B_{\epsilon} ; \mathbb{R}^{2}\right)$, or $\mathcal{C}=\mathcal{C}_{\theta}$ and a fixed $V \in C^{0}\left(\overline{B_{1}} \backslash B_{\epsilon} ; \mathbb{R}^{2}\right)$, and given $n \geq 1$, we introduce

$$
\mathcal{C}_{n}=\left\{u \in \mathcal{C}: D u \cdot V \geq \frac{1}{n} \quad \text { and } \quad\|u\|_{C^{2, \alpha}\left(\overline{B_{1}} \backslash B_{\epsilon}\right)} \leq n\right\}
$$

Suppose $\mathcal{C}_{N_{0}} \neq \emptyset$, for some $N_{0} \geq 1$. Given any $1 \leq p<\infty$, the functional $F_{p}: \mathcal{C}_{n} \rightarrow \mathbb{R}, n \geq N_{0}$, defined by

$$
u \rightarrow F_{p}(u)=\int_{B_{1} \backslash B_{\epsilon}}\left(\frac{|D u|^{2}+|V|^{2}}{D u \cdot V}\right)^{p} \mathrm{~d} x
$$

is strictly convex, continuous, and attains its infimum on $\mathcal{C}_{n}$ at a unique minimizer. If the unique minimizer, $u$, lies in int $\left(\mathcal{C}_{n}\right)^{5}$, then it satisfies the associated Euler-Lagrange equation

$$
\operatorname{div}\left(\left(\frac{|D u|^{2}+|V|^{2}}{D u \cdot V}\right)^{p-1}\left(\frac{2 D u}{D u \cdot V}-\frac{|D u|^{2}+|V|^{2}}{(D u \cdot V)^{2}} V\right)\right)=0 \quad \text { in } B_{1} \backslash \overline{B_{\epsilon}},
$$

and in the case $\mathcal{C}=\mathcal{C}_{\theta}$, the additional boundary condition

$$
\left(\frac{2 D u}{D u \cdot V}-\frac{|D u|^{2}+|V|^{2}}{(D u \cdot V)^{2}} V\right) \cdot \frac{x}{|x|}=0 \quad \text { on } C_{\epsilon} .
$$

Conversely, if there exists a solution to (4.1) (and (4.2) in case $\mathcal{C}=\mathcal{C}_{\theta}$ ) which lies in $\mathcal{C} \cap$ $\left\{D u \cdot V>0\right.$ on $\left.\overline{B_{1}} \backslash B_{\epsilon}\right\}$, then, for some $N \geq 1$, this is the unique minimizer of $F_{p}$ in $\mathcal{C}_{n}$, for any $n \geq N$. Consequently this $u$ is also the unique minimizer of $F_{p}$ in $\mathcal{C} \cap\left\{D u \cdot V>0\right.$ on $\left.\overline{B_{1}} \backslash B_{\epsilon}\right\}$.

\footnotetext{
${ }^{4}$ The space $\quad C^{\infty}\left(\overline{B_{1}} \backslash B_{\epsilon} ; \mathbb{R} / 2 \pi \mathbb{Z}\right) \quad$ is $\quad$ defined $\quad$ as $\quad\left\{u \in C^{1}\left(\overline{B_{1}} \backslash B_{\epsilon} ; \mathbb{R} / 2 \pi \mathbb{Z}\right): D u \in C^{\infty}\left(\overline{B_{1}} \backslash B_{\epsilon} ; \mathbb{R}^{2}\right)\right\} . \quad$ Similarly $C^{2, \alpha}\left(\overline{B_{1}} \backslash B_{\epsilon} ; \mathbb{R} / 2 \pi \mathbb{Z}\right)=\left\{u \in C^{1}\left(\overline{B_{1}} \backslash B_{\epsilon} ; \mathbb{R} / 2 \pi \mathbb{Z}\right): D u \in C^{1, \alpha}\left(\overline{B_{1}} \backslash B_{\epsilon} ; \mathbb{R}^{2}\right)\right\}$.

${ }^{5}$ The interior is formed relative to $\mathcal{C}_{\psi}$ or $\mathcal{C}_{\theta}$ with the $C^{2, \alpha}$ topology, respectively.
} 
For the proof of Proposition 4.1 we shall need the following lemma.

Lemma 4.2. For any $1 \leq p<\infty$, and any $A>0$, the function $G_{p}[A]:(0, \infty) \times \mathbb{R} \rightarrow \mathbb{R}_{+}$, given by

$$
(x, y) \rightarrow\left(\frac{A}{x}+\frac{x}{A}+\frac{x}{A}\left(\frac{y}{x}\right)^{2}\right)^{p}
$$

is convex. Furthermore,

$$
G_{p}[A](x, y)-\frac{2 A^{4}}{\left(A^{2}+M^{2}\right)^{3}}\left(x^{2}+y^{2}\right)
$$

is convex on $B_{M}=\left\{(x, y): x^{2}+y^{2}<M^{2}\right\}$.

Proof. The function $x \rightarrow \frac{A}{x}+\frac{x}{A}$ is strictly convex and positive valued on $(0, \infty) \times \mathbb{R}$. The map $(x, y) \rightarrow \frac{1}{A} \frac{y^{2}}{x}$ is convex and positive on $(0, \infty) \times \mathbb{R}$. Indeed, its Hessian has eigenvalues 0 and $\frac{2}{A} \frac{x^{2}+y^{2}}{x^{3}}$. The sum of two convex (and positive valued) functions is convex (and positive valued), and the composition of it with $z \rightarrow z^{p}$, a monotonically increasing and convex function on $(0, \infty)$, results in a convex (positive valued) function.

To establish the second assertion, we compute lower bounds for $D^{2} G_{p}[A]$. It is a fact that the lowest eigenvalue of a symmetric positive definite matrix is bounded below by the quotient of the determinant over the trace. We compute that for $p \geq 1$,

$$
\frac{\operatorname{det}\left(D^{2} G_{p}[A]\right)}{\operatorname{tr}\left(D^{2} C_{p}[A]\right)}>\frac{4 p}{p+1} G_{p}[A] \frac{A^{4}}{\left(A^{2}+x^{2}+y^{2}\right)^{3}} \geq 4 \frac{A^{4}}{\left(A^{2}+x^{2}+y^{2}\right)^{3}} .
$$

In particular, on the ball $B_{M}=\left\{(x, y): x^{2}+y^{2}<M^{2}\right\}$ we have

$$
D^{2} G_{p}[A](x, y)>\frac{4 A^{4}}{\left(A^{2}+M^{2}\right)^{3}} I .
$$

This immediately leads to the second assertion of the lemma.

We are now ready for the proof of Proposition 4.1.

Proof. Given $u \in \mathcal{C}_{n}$, we define

$$
P_{V}(D u)=D u \cdot \frac{V}{|V|}, \text { and } P_{V^{\perp}}(D u)=D u \cdot \frac{J V}{|V|} .
$$

Then

$$
\begin{aligned}
\left(\frac{|D u|^{2}+|V|^{2}}{D u \cdot V}\right)^{p} & =\left(\frac{|V|}{P_{V}(D u)}+\frac{P_{V}(D u)}{|V|}+\frac{P_{V}(D u)}{|V|}\left(\frac{P_{V^{\perp}}(D u)}{P_{V}(D u)}\right)^{2}\right)^{p} \\
& =G_{p}[|V|]\left(P_{V}(D u), P_{V^{\perp}}(D u)\right) .
\end{aligned}
$$

Note that $\mathcal{C}_{N_{0}} \neq \emptyset$ implies $\inf |V|>0$. On $\mathcal{C}_{n},\left|P_{V}(D u)\right|^{2}+\left|P_{V^{\perp}}(D u)\right|^{2} \leq n^{2}$, and therefore for any $u, v \in \mathcal{C}_{n}$, $n \geq N_{0}$, and any $\tau \in[0,1]$

$$
\begin{aligned}
G_{p}[|V|] & \left(P_{V}(D(\tau u+(1-\tau) v)), P_{V^{\perp}}(D(\tau u+(1-\tau) v))\right) \\
\leq & \tau G_{p}[|V|]\left(P_{V}(D u), P_{V^{\perp}}(D u)\right)+(1-\tau) G_{p}[|V|]\left(P_{V}(D v), P_{V^{\perp}}(D v)\right) \\
& -\tau(1-\tau) K|D(u-v)|^{2}
\end{aligned}
$$


with

$$
K=\frac{2 \inf |V|^{4}}{\left(n^{2}+\sup |V|^{2}\right)^{3}}>0 .
$$

For $u, v \in \mathcal{C}_{n}$, and $\tau \in[0,1]$, we thus get

$$
F_{p}(\tau u+(1-\tau) v) \leq \tau F_{p}(u)+(1-\tau) F_{p}(v)-\tau(1-\tau) K \int_{B_{1} \backslash B_{\epsilon}}|D(u-v)|^{2} \mathrm{~d} x,
$$

and so $F_{p}$ is strictly convex on $\mathcal{C}_{n}$. In regards to continuity, let $u_{m}$ be a sequence in $\mathcal{C}_{n}$ with $u_{m} \rightarrow u$ in the $C^{1}$ topology. Then the functions

$$
x \rightarrow G_{p}[|V|]\left(P_{V}\left(D u_{m}\right), P_{V^{\perp}}\left(D u_{m}\right)\right)(x)
$$

are measurable, non negative, uniformly bounded, and converge pointwise to the function

$$
x \rightarrow G_{p}[|V|]\left(P_{V}(D u), P_{V^{\perp}}(D u)\right)(x) .
$$

Thanks to the Lebesgue Dominated Convergence Theorem, this implies

$$
\lim F_{p}\left(u_{m}\right)=F_{p}(u) .
$$

Since $\mathcal{C}_{n}$ is compact with respect to the $C^{1}$ topology, the $C^{1}$ continuity of $F_{p}$ implies the existence of a minimizer. The convexity of $\mathcal{C}_{n}$ and the strict convexity of $F_{p}$ yields the uniqueness of the minimizer. A computation shows that for any $u \in \mathcal{C}_{n}, F_{p}$ is Gâteaux-differentiable at $u$, and its differential is given by

$$
\left\langle D F_{p}(u), h\right\rangle=\int_{B_{1} \backslash B_{\epsilon}} p\left(\frac{|D u|^{2}+|V|^{2}}{D u \cdot V}\right)^{p-1}\left(\frac{2 D u}{D u \cdot V}-\frac{|D u|^{2}+|V|^{2}}{(D u \cdot V)^{2}} V\right) \cdot D h \mathrm{~d} x,
$$

for $h \in C^{1}$. Note that $u \in \mathcal{C}_{n}$ is the unique minimizer if and only if for all $v \in \mathcal{C}_{n}$ there holds

$$
\left\langle D F_{p}(u), v-u\right\rangle \geq 0 .
$$

If the minimizer lies in the interior of $\mathcal{C}_{n},(4.3)$ implies

$$
\left\langle D F_{p}(u), h\right\rangle=0
$$

for all $h \in C^{2, \alpha} \cap\left\{h=0\right.$ on $C_{\epsilon}$ and $\left.C_{1}\right\}$, if $\mathcal{C}=\mathcal{C}_{\psi}$, and for all $h \in C^{2, \alpha} \cap\left\{h=0\right.$ on $\left.C_{1}\right\}$, if $\mathcal{C}=\mathcal{C}_{\theta}$; in other words, $u$ satisfies the Euler-Lagrange equation (4.1) (or (4.1) and (4.2) when $\mathcal{C}=\mathcal{C}_{\theta}$ ). Conversely, if $w \in \mathcal{C} \cap\left\{D u \cdot V>0\right.$ on $\left.\overline{B_{1}} \backslash B_{\epsilon}\right\}$ satisfies (4.1) (and (4.2) if $\mathcal{C}=\mathcal{C}_{\theta}$ ), then, for some $N$, it lies in $\mathcal{C}_{n}$ for all $n \geq N$, and it satisfies $\left\langle D F_{p}(w), v-w\right\rangle=0$ (in particular $\geq 0$ ) for all $v \in \mathcal{C}_{n} ; w$ is thus the unique minimizer of $F_{p}$ in $\mathcal{C}_{n}$ for any $n \geq N$. It follows immediately that $w$ is a minimizer of $F_{p}$ in $\mathcal{C} \cap\left\{D u \cdot V>0\right.$ on $\left.\overline{B_{1}} \backslash B_{\epsilon}\right\}$. The uniqueness of this minimizer follows from the strict convexity of $F_{p}$ on $\mathcal{C}_{n}$ for any $n$.

Corollary 4.3. A global $C^{2, \alpha}$ minimizer $(\psi, \theta)$ of $I_{p}$, subject to $\psi=-\log 2$ at $|x|=\epsilon, \psi=0$ and $\theta=\arg$ at $|x|=1$, and $\operatorname{det}(D \psi, D \theta)>0$ on $\overline{B_{1}} \backslash B_{\epsilon}$, satisfies

$$
\operatorname{div}\left(\left(\frac{|D \psi|^{2}+|D \theta|^{2}}{\operatorname{det}(D \psi, D \theta)}\right)^{p}\left(\frac{2 D \psi}{|D \psi|^{2}+|D \theta|^{2}}+\frac{J D \theta}{\operatorname{det}(D \psi, D \theta)}\right)\right)=0,
$$

and

$$
\operatorname{div}\left(\left(\frac{|D \psi|^{2}+|D \theta|^{2}}{\operatorname{det}(D \psi, D \theta)}\right)^{p}\left(\frac{2 D \theta}{|D \psi|^{2}+|D \theta|^{2}}-\frac{J D \psi}{\operatorname{det}(D \psi, D \theta)}\right)\right)=0 .
$$

Furthermore,

$$
\left(\frac{|D \psi|^{2}+|D \theta|^{2}}{\operatorname{det}(D \psi, D \theta)} J D \psi-2 D \theta\right) \cdot \frac{x}{|x|}=0 \quad \text { on }\{|x|=\epsilon\}
$$




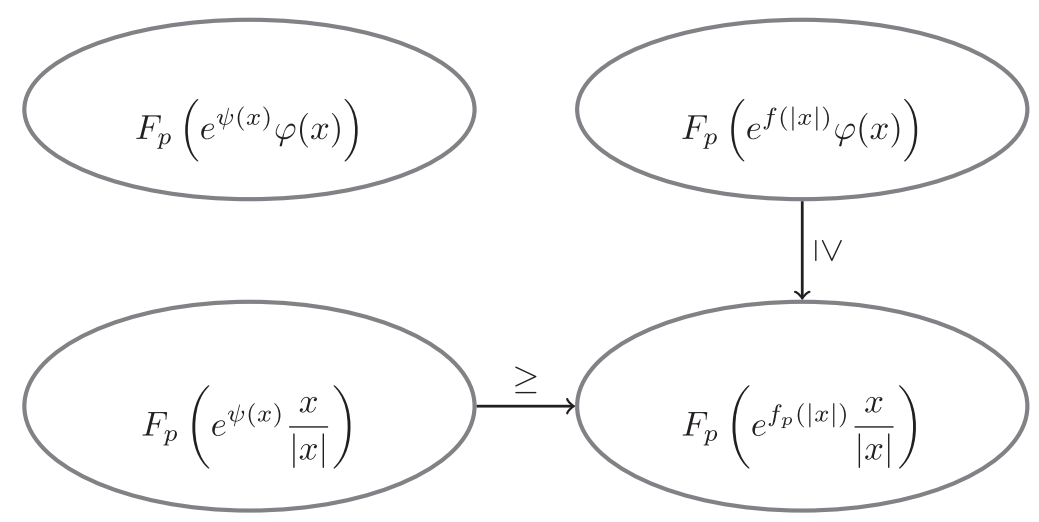

Figure 1. Illustration of the conclusions of Corollary 4.4.

Proof. The $\psi$ component of this global minimizer automatically lies $\operatorname{in} \operatorname{int}\left(\mathcal{C}_{n}\right)$ with $\mathcal{C}=\mathcal{C}_{\psi}$ and $V=-J D \theta$ for some $n$, and it is a minimizer of $F_{p}$ in $\mathcal{C}_{n}$. The first equation of this corollary is now simply the Euler-Lagrange (4.1) for such a minimizer. Similarly, the $\theta$ component of this global minimizer lies $\operatorname{in} \operatorname{int}\left(\mathcal{C}_{n}\right)$ with $\mathcal{C}=\mathcal{C}_{\theta}$ and $V=J D \psi$ for some $n$, and is a minimizer of $F_{p}$ in $\mathcal{C}_{n}$. The two last equations of this corollary are simply the Euler-Lagrange (4.1) and the boundary condition (4.2) satisfied by such a minimizer.

Corollary 4.4. Let $f_{p}$ be the function introduced in Proposition 3.1. The transformation $x \rightarrow f_{p}(|x|) \frac{x}{|x|}$, or rather the function pair $\left(f_{p}(|x|), \arg (x)\right)$ satisfies the three Euler-Lagrange equations from Corollary 4.3. As a consequence

$$
I_{p}\left(f_{p}(|x|) \frac{x}{|x|}\right) \leq I_{p}\left(\psi(x) \frac{x}{|x|}\right)
$$

for any $\psi \in \mathcal{C}_{\psi} \cap\left\{D \psi(x) \cdot \frac{x}{|x|}>0\right.$ on $\left.\overline{B_{1}} \backslash B_{\epsilon}\right\}$. The last two Euler-Lagrange equations from Corollary 4.3 are actually satisfied by any pair $(f(|x|), \arg (x))$, with $f \in\left\{f \in C^{2, \alpha}([\epsilon, 1]): f^{\prime}>0, f(\epsilon)=-\log 2, f(1)=0\right\}$. As a consequence

$$
I_{p}\left(f_{p}(|x|) \frac{x}{|x|}\right) \leq I_{p}\left(f(|x|) \frac{x}{|x|}\right) \leq I_{p}(f(|x|) \phi(x)),
$$

for any $\phi(x)=\left(\cos (\theta(x)), \sin (\theta(x))^{t}\right.$, with $\theta \in \mathcal{C}_{\theta} \cap\left\{D \theta \cdot J \frac{x}{|x|}>0\right.$ on $\left.\overline{B_{1}} \backslash B_{\epsilon}\right\}$ and any $f \in$ $\left\{f \in C^{2, \alpha}([\epsilon, 1]): f^{\prime}>0, f(\epsilon)=-\log 2, f(1)=0\right\}$.

Proof. Direct calculations verify that the first Euler-Lagrange equation from Corollary 4.3 is satisfied by the pair $\left(f_{p}(|x|), \arg (x)\right)$, and that the last two Euler-Lagrange equations from Corollary 4.3 are satisfied by any pair $(f(|x|), \arg (x))$, with $f \in\left\{f \in C^{2, \alpha}([\epsilon, 1]): f^{\prime}>0, f(\epsilon)=-\log 2, f(1)=0\right\}$. The inequality (4.4) now follows immediately from the last statement in Proposition 4.1 in the case $\mathcal{C}=\mathcal{C}_{\psi}$ and $V=-J D \arg (x)=\frac{1}{|x|} \frac{x}{|x|}$. The first inequality in (4.5) is a direct consequence of (4.4). The second inequality follows from the last statement in Proposition 4.1 in the case $\mathcal{C}=\mathcal{C}_{\theta}$ and $V=J D f(|x|)=f^{\prime}(|x|) J \frac{x}{|x|}$.

\section{Optimal ClOAKS FOR SIMPLY CONNECTED DOMAINS}

So far our study has focused on the situation where the cloaks are constructed from diffeomorphisms of the classical annulus $\overline{B_{1}} \backslash B_{\epsilon}$ to the classical annulus $\overline{B_{1}} \backslash B_{\frac{1}{2}}$, and the corresponding push-forwards of the identity matrix. In a more general setting, one could consider instead three simply connected domains, $\omega_{\epsilon} \subset \omega_{\frac{1}{2}} \subset \Omega$ 


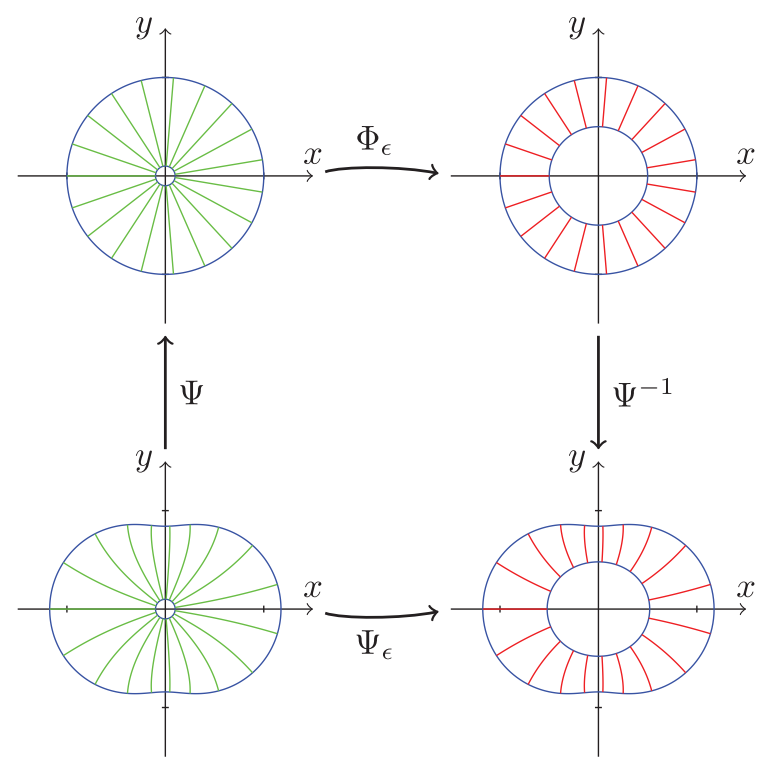

Figure 2. Cloaking by mapping where $\Omega=\sinh \left(B_{1}\right)$, with $\epsilon=1 / 10$.

containing the origin (where $\omega_{\epsilon}$ is comparable to $B_{\epsilon}$ ) and a bijective diffeomorphism $\Psi_{\epsilon}: \bar{\Omega} \backslash \omega_{\epsilon} \rightarrow \bar{\Omega} \backslash \omega_{\frac{1}{2}}$, such that $\Psi_{\epsilon}=I d$ on $\partial \Omega$ and $\Psi_{\epsilon}\left(\partial \omega_{\epsilon}\right)=\partial \omega_{\frac{1}{2}}$. As before, the material parameters of the cloak would be the push-forward of $I$ by $\Psi_{\epsilon}$. Any smooth globally minimizing transformation would still satisfy the Euler-Lagrange equations of Corollary 4.3, if we continue to use the energy $I_{p}$. We note that the convexity properties established in Proposition 4.1 suggest a possible numerical strategy to search for a minimizer, namely: alternatingly performing a minimization with respect to angle (for fixed logarithmic amplitude) and a minimization with respect to logarithmic amplitude (for fixed angle).

The goal of this section is to show that for general geometries one should (naturally) not expect the optimal transformations to be radial. As we demonstrate this, we also derive a process for the construction of optimal transformations (based on a slightly revised energy). Suppose $\Omega$ is a bounded, smooth, simply connected domain containing the origin. Due to the Riemann Mapping Theorem, there exists a unique (complex) analytic map $\Psi$ such that $\Psi(0)=0, D \Psi(0)=a I$ for some $a>0$ and $\Psi$ is a one-to-one mapping from $\bar{\Omega}$ onto $\overline{B_{1}}$. By the maximum modulus principle $\min \{|x|: x \in \bar{\Omega}\} \leq 1 / a \leq \max \{|x|: x \in \bar{\Omega}\}$. Set $\omega_{\epsilon}=\Psi^{-1}\left(B_{\epsilon}\right)$, and $\omega_{\frac{1}{2}}=\Psi^{-1}\left(B_{\frac{1}{2}}\right)$. By construction, $0 \in \omega_{\epsilon} \subset \omega_{\frac{1}{2}} \subset \Omega$. Provided $\epsilon$ is small enough, $\omega_{\epsilon}$ is approximately $B_{\frac{\epsilon}{a}}$, in the sense that

$$
\forall x \in C_{\epsilon} \quad\left|\Psi^{-1}(x)-\frac{x}{a}\right| \leq \frac{1}{2} \frac{\max }{B_{1 / 2}}\left|D^{2} \Psi^{-1}\right| \epsilon^{2} .
$$

Given $\Phi_{\epsilon} \in C^{1}\left(\overline{B_{1}} \backslash B_{\epsilon} ; \overline{B_{1}} \backslash B_{\frac{1}{2}}\right)$ a (possibly optimal) bijective diffeomorphism with $\left.\Phi_{\epsilon}\right|_{C_{1}}=I d$, and $\Phi_{\epsilon}\left(C_{\epsilon}\right)=$ $C_{\frac{1}{2}}$, we define

$$
\Psi_{\epsilon}:=\Psi^{-1} \circ \Phi_{\epsilon} \circ \Psi
$$

Figure 2 shows some of the "rays" of the map $\Psi_{\epsilon}\left(\Phi_{\epsilon}\right.$ being radial) in the case $\Psi^{-1}=\sinh , \Omega=\sinh \left(B_{1}\right)$, $\omega_{\frac{1}{2}}=\sinh \left(B_{\frac{1}{2}}\right)$ and $\omega_{\epsilon}=\sinh \left(B_{\epsilon}\right)$. The green curves on the left are mapped to proper subsets of themselves, shown as red curves on the right. Clearly the transformation $\Psi_{\epsilon}$ is no longer radial.

For any $x \in \partial \Omega, \Psi(x)$ lies on $C_{1}$, and thus $\Phi_{\epsilon} \circ \Psi(x)=\Psi(x)$. It follows that $\Psi_{\epsilon}(x)=x$, in other words: $\Psi_{\epsilon}=I d$ on $\partial \Omega$. Similarly, we obtain that $\Psi_{\epsilon}\left(\partial \omega_{\epsilon}\right)=\partial \omega_{\frac{1}{2}} \cdot\left(\Psi_{\epsilon}\right)_{\star}[I]$ therefore produces an approximate cloak 
(with same approximate invisibility as that of $\left(\Phi_{\epsilon}\right)_{\star}[I]$ ). From composition of transformations we obtain

$$
\left(\Psi_{\epsilon}\right)_{\star}[I]=\left(\Psi^{-1}\right)_{\star}\left[\left(\Phi_{\epsilon}\right)_{\star}\left[\Psi_{\star}[I]\right]\right] .
$$

Lemma 5.1. There holds

$$
\operatorname{trace}\left(\Psi_{\epsilon}\right)_{\star}[I]=\left(\operatorname{trace}\left(\Phi_{\epsilon}\right)_{\star}[I]\right) \circ \Psi .
$$

Proof. Since $\Psi$ is conformal, $D \Psi=\gamma Q$ with $\gamma$ a positive scalar and $Q$ an orthogonal matrix. We are in 2 d, and so this implies

$$
\Psi_{\star}[I](y)=\frac{(D \Psi)(D \Psi)^{T}}{|\operatorname{det} D \Psi|} \circ \Psi^{-1}(y)=I .
$$

Similarly,

$$
\begin{aligned}
\left(\Psi^{-1}\right)_{\star}[A](x) & =\frac{\left(D \Psi^{-1}\right) A\left(D \Psi^{-1}\right)^{T}}{\left|\operatorname{det} D \Psi^{-1}\right|} \circ \Psi(x) \\
& =Q^{T}(x) A(\Psi(x)) Q(x),
\end{aligned}
$$

where we have used that $\left(D \Psi^{-1}\right)(\Psi(x))=(D \Psi)^{-1}(x)=\frac{1}{\gamma} Q^{T}(x)$. In summary, we conclude that $\left(\Psi_{\epsilon}\right)_{\star}[I]$ is given by the formula

$$
\left(\Psi_{\epsilon}\right)_{\star}[I](x)=Q^{T}(x)\left(\Phi_{\epsilon}\right)_{\star}[I](\Psi(x)) Q(x),
$$

and the statement about the traces follows.

If $\Phi_{\epsilon}$ is a transformation which minimizes the anisotropy of $\left(\Phi_{\epsilon}\right)_{\star}[I]$, using the measure $I_{p}$ for some $1 \leq p<\infty$, then it follows immediately from Lemma 5.1 above that $\Psi_{\epsilon}$ minimizes anistropy of $\left(\Psi_{\epsilon}\right)_{\star}[I]$, using the slightly modified measure

$$
\tilde{I}_{p}\left(\Psi_{\epsilon}\right)=\int_{\Omega \backslash \omega_{\epsilon}}\left(\operatorname{trace}\left(\Psi_{\epsilon}\right)_{*}[I]\right)^{p}\left(\Psi_{\epsilon}(x)\right)|\operatorname{det} \Psi(x)| \mathrm{d} x .
$$

A similar statement holds for $p=\infty$. In that case there is no change in the measure of anisotropy.

Acknowledgements. The research of MSV was partially supported by NSF Grant DMS-12-11330. Part of this work was carried out while MSV was visiting the University of Copenhagen and the Danish Technical University. This visit was made possible through support from the Nordea Foundation and the Otto Mønsted Foundation. This study also contributes to the IdEx Université de Paris ANR-18-IDEX-0001.

\section{REFERENCES}

[1] G.V. Alekseev, Analysis of a two-dimensional thermal cloaking problem on the basis of optimization. Comput. Math. Math. Phys. 58 (2018) 478-492.

[2] H. Ammari, J. Garnier, V. Jugnon, H. Kang, H. Lee and M. Lim, Enhancement of near-cloaking. Part III: numerical simulations, statistical stability, and related questions. In: Multi-scale and High-contrast PDE. From Modelling, to Mathematical Analysis, to Inversion. Proceedings of the Conference, University of Oxford, UK, June 28-July 1, 2011. American Mathematical Society (AMS), Providence, RI (2012) 1-24.

[3] H. Ammari, H. Kang, H. Lee and M. Lim, Enhancement of near cloaking using generalized polarization tensors vanishing structures. I: the conductivity problem. Commun. Math. Phys. 317 (2013) 253-266.

[4] A. Greenleaf, M. Lassas and G. Uhlmann, On nonuniqueness for Calderón's inverse problem. Math. Res. Lett. 10 (2003) 685-693.

[5] A. Greenleaf, Y. Kurylev, M. Lassas and G. Uhlmann, Invisibility and inverse problems. Bull. Am. Math. Soc. New Ser. 46 (2009) 55-97.

[6] R. Griesmaier and M.S. Vogelius, Enhanced approximate cloaking by optimal change of variables. Inverse Prob. 30 (2014) 17.

[7] H. Heumann and M.S. Vogelius, Analysis of an enhanced approximate cloaking scheme for the conductivity problem. Asymp. Anal. 87 (2014) 223-246.

[8] R.V. Kohn, H. Shen, M.S. Vogelius and M.I. Weinstein, Cloaking via change of variables in electric impedance tomography. Inverse Prob. 24 (2008) 21. 
[9] G.W. Milton and N.-A.P. Nicorovici, On the cloaking effects associated with anomalous localized resonance. Proc. Math. Phys. Eng. Sci. 462 (2006) 3027-3059.

[10] J.B. Pendry, D. Schurig and D.R. Smith, Controlling electromagnetic fields. Science 312 (2006) $1780-1782$.

[11] C.-W. Qiu, L. Hu, B. Zhang, B.-I. Wu, S.G. Johnson and J.D. Joannopoulos, Spherical cloaking using nonlinear transformations for improved segmentation into concentric isotropic coatings. Opt. Express 17 (2009) 13467-13478.

\section{Subscribe to Open (S20) A fair and sustainable open access model}

This journal is currently published in open access under a Subscribe-to-Open model (S2O). S2O is a transformative model that aims to move subscription journals to open access. Open access is the free, immediate, online availability of research articles combined with the rights to use these articles fully in the digital environment. We are thankful to our subscribers and sponsors for making it possible to publish this journal in open access, free of charge for authors.

\section{Please help to maintain this journal in open access!}

Check that your library subscribes to the journal, or make a personal donation to the S2O programme, by contacting subscribers@edpsciences.org

More information, including a list of sponsors and a financial transparency report, available at: https://www. edpsciences.org/en/maths-s2o-programme 